\title{
Effect of water vapour absorption on hydroxyl temperatures measured from Svalbard
}

\author{
Joshua M. Chadney, Daniel K. Whiter, and Betty S. Lanchester \\ Department of Physics and Astronomy, University of Southampton, Southampton, SO17 1BJ, UK \\ Correspondence to: J. M. Chadney (j.m.chadney@soton.ac.uk)
}

Received: 12 December 2016 - Revised: 28 February 2017 - Accepted: 1 March 2017 - Published: 24 March 2017

\begin{abstract}
We model absorption by atmospheric water vapour of hydroxyl airglow emission using the HIghresolution TRANsmission molecular absorption database (HITRAN2012). Transmission coefficients are provided as a function of water vapour column density for the strongest $\mathrm{OH}$ Meinel emission lines in the (8-3), (5-1), (9-4), (8-4), and (6-2) vibrational bands. These coefficients are used to determine precise $\mathrm{OH}(8-3)$ rotational temperatures from spectra measured by the High Throughput Imaging Echelle Spectrograph (HiTIES), installed at the Kjell Henriksen Observatory (KHO), Svalbard. The method described in this paper also allows us to estimate atmospheric water vapour content using the HiTIES instrument.
\end{abstract}

Keywords. Atmospheric composition and structure (airglow and aurora; middle atmosphere - composition and chemistry; pressure density and temperature)

\section{Introduction}

A layer of excited hydroxyl molecules near the mesopause is responsible for strong airglow emission that can be detected over a wide wavelength range. First identified by Meinel (1950a, b), this emission is produced by molecular vibration-rotation transitions. It is possible to derive rotational $\mathrm{OH}$ temperatures by determining ratios between emission lines. If one considers that the lower rotational and vibrational states have undergone enough collisions to be in local thermodynamic equilibrium (LTE), then the neutral atmospheric temperature can be assumed to match the rotational hydroxyl temperature. This method has been used by many authors to calculate mesospheric temperatures (e.g. French et al., 2000; Phillips et al., 2004; Suzuki et al., 2010; Holmen et al., 2014a). In this study, we use the same nomenclature as Phillips et al. (2004) to describe hydroxyl emissions, as detailed in Appendix A of that paper.

$\mathrm{OH}$ temperatures have been derived in previous works using different vibrational bands, depending on the wavelength range of the spectroscope in use. French et al. (2000) and Holmen et al. (2014a) measured the $\mathrm{OH}(6-2)$ band, Phillips et al. (2004) compared $\mathrm{OH}(6-2)$ band observations with measurements from $\mathrm{OH}(8-3)$, and Suzuki et al. (2010) made use of the $\mathrm{OH}(8-4)$ band. In this study, we use HiTIES, the High Throughput Imaging Echelle Spectrograph (Chakrabarti et al., 2001), part of the Spectrographic Imaging Facility (SIF), located at the Kjell Henriksen Observatory (KHO), Svalbard $\left(78.148^{\circ} \mathrm{N}, 16.043^{\circ} \mathrm{E}\right)$, to record hydroxyl emission line intensities. We use the HiTIES filter panel with a bandpass from 728 to $740 \mathrm{~nm}$, allowing measurement of the $\mathrm{OH}(8-3)$ band. The emission peak altitude is dependent on vibrational level (von Savigny et al., 2012), the mean thickness of the $\mathrm{OH}$ layer being about $8 \mathrm{~km}$ (Baker and Stair, 1988) with higher vibrational levels located at the top of the layer and vice versa. Due to the spread in altitude of the different vibrational excited states, there is expected to be a difference in the rotational temperatures derived from emission from different vibrational bands. The temperature difference between bands is thought to vary from a few kelvin to up to $20 \mathrm{~K}$ between bands with the largest difference in upper vibrational state $v^{\prime}$ (Cosby and Slanger, 2007; von Savigny et al., 2012; Noll et al., 2015, 2016).

A difficulty in determining accurate temperatures from $\mathrm{OH}$ lines is the absorption of this emission by other atmospheric species, in particular water vapour. Phillips et al. (2004) found that water vapour absorption decreases the intensity of the $\mathrm{OH}(8-3) \mathrm{P}_{1}(4)$ line by $2.3 \%$, affecting the temperature derived using a ratio between this line and other $\mathrm{P}$ branch lines in the (8-3) band by up to $17 \mathrm{~K}$. This calculation 

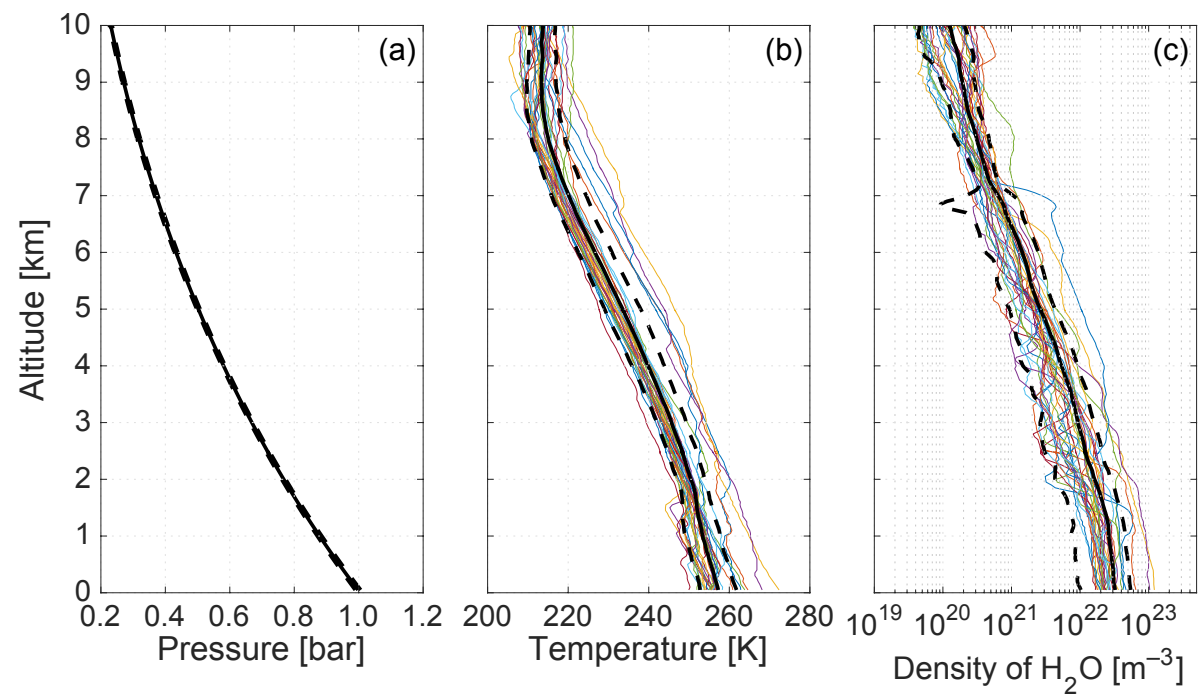

Figure 1. Atmospheric profiles used in the water vapour absorption model, measured by radiosonde over Ny-Ålesund, Svalbard, from Maturilli and Kayser (2016). Panel (a) shows the pressure as a function of altitude, panel (b) shows the temperature, and panel (c) shows the number density of $\mathrm{H}_{2} \mathrm{O}$. The coloured lines each represent a daily observation during the month of December 2003; the mean profiles from this month are plotted in solid black lines with 1 standard deviation from the mean shown in dashed black lines.

was carried out only for average winter conditions at Davis Station, Antarctica; however, this absorption is dependent on the concentration of water vapour in the atmosphere, which is highly variable, and will also be on average higher at lower latitudes.

To quantify the effects of atmospheric absorption on $\mathrm{OH}$ emission, Espy and Hammond (1995) modelled atmospheric absorption of a number of species (such as $\mathrm{H}_{2} \mathrm{O}, \mathrm{CO}_{2}$, and $\mathrm{O}_{3}$ ), as well as $\mathrm{OH}$ line profiles to provide transmission coefficients for $\mathrm{OH}$ Meinel rotational lines in a number of different vibrational bands. These coefficients are provided for four different atmospheric conditions: mid- and high-latitude summer, and mid- and high-latitude winter. Unfortunately, their table giving transmission coefficients for the $\mathrm{OH}(8-3)$ (Table 6) is a repeat of the $\mathrm{OH}(6-2)$ table (Table 5) and is thus erroneous.

In this work, we model water vapour absorption in the atmosphere and provide transmission of various $\mathrm{OH}$ Meinel band lines as a function of the concentration of atmospheric $\mathrm{H}_{2} \mathrm{O}$. Whereas Espy and Hammond (1995) provided transmission values for four discrete concentrations of water vapour, here we provide the transmission as a continuous function of water vapour concentration. This paper is organised as follows: Sect. 2 describes the instruments, models, and methods used in this study; Sect. 3 provides the transmission coefficients for $\mathrm{OH}$ lines; and Sect. 4 shows the effect of water vapour absorption on temperatures derived from $\mathrm{OH}$ lines in the (8-3) vibrational band measured by HiTIES.

\section{Methods}

\subsection{Modelling water vapour absorption}

The transmission of emission at a given wavelength $\lambda$ as it passes through the atmosphere can be determined using the Beer-Lambert law:

$\tau(\lambda)=\int_{0}^{z \mathrm{OH}} \sigma_{\mathrm{H}_{2} \mathrm{O}}^{\mathrm{abs}}(\lambda, z) n_{\mathrm{H}_{2} \mathrm{O}}(z) \mathrm{d} z$,

where $\tau$ is the optical depth, $\sigma_{\mathrm{H}_{2} \mathrm{O}}^{\text {abs }}$ is the absorption cross section of $\mathrm{H}_{2} \mathrm{O}, n_{\mathrm{H}_{2} \mathrm{O}}$ is the number density of $\mathrm{H}_{2} \mathrm{O}$, and $z$ is the altitude. This expression gives the optical depth over a column of atmosphere between $z=z_{\mathrm{OH}}$, the altitude at which the hydroxyl emission occurs, and $z=0$, where the instruments measuring the emission are located. Equation (1) is valid for a vertical beam, where there is only extinction in the beam (i.e. no emission within the beam or scattering into the beam). The transmission coefficient can then be obtained from

$T_{\lambda}=\exp (-\tau(\lambda))$.

To determine the absorption cross section, we use the latest version of the HIgh-resolution TRANsmission molecular absorption database, HITRAN2012 (Rothman et al., 2013). This gives the $\mathrm{H}_{2} \mathrm{O}$ absorption cross section $\sigma_{\mathrm{H}_{2} \mathrm{O}}^{\mathrm{abs}}$ for given temperature and pressure conditions, used to determine line broadening. Therefore to calculate transmission coefficients, we need atmospheric profiles of pressure $p(z)$, temperature $T(z)$, and water vapour number density $n_{\mathrm{H}_{2} \mathrm{O}}(z)$. These are 


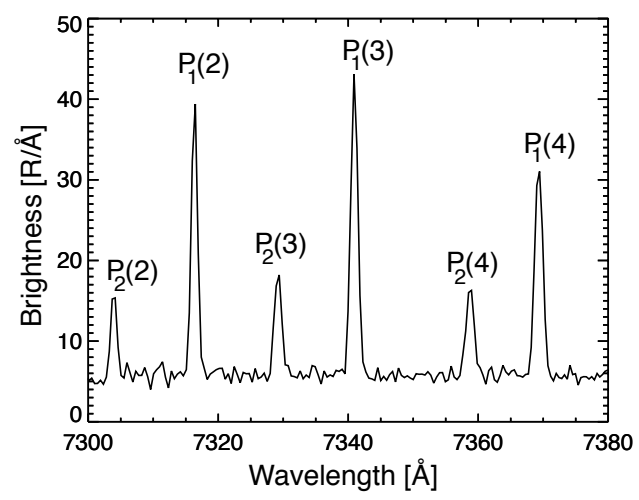

Figure 2. Example clear-sky HiTIES spectrum, showing labelled OH emission lines. Taken on 23 December 2003 at 22:30 UT, with an integration time of $120 \mathrm{~s}$.

obtained from radiosonde measurements over Ny-Ålesund, Svalbard by Maturilli and Kayser (2016). Daily observations have been made since 1993.

Since in Sect. 4 we take a week of $\mathrm{OH}$ line intensity measurements from December 2003 as an example to determine temperatures, we have used atmospheric profiles from this month from the Ny-Ålesund dataset. These are plotted in Fig. 1, where the thin coloured lines each represent a daily observation from December 2003 (all days of this month are included); the thick black lines are the mean of all the daily values and so represent a monthly average during winter.

Water vapour is principally located in the troposphere, at altitudes less than about $10 \mathrm{~km}$. Therefore, we need only the atmospheric profiles below this altitude. To take into account varying water vapour column densities, the monthly mean profile from Fig. 1c is scaled such that the integrated column density matches the required value. We have tested using a selected daily profile instead of the monthly mean and obtain little difference in the results.

Atmospheric water vapour content is often provided as precipitable water vapour (PWV), normally expressed in millimetres, which is the height of a column of liquid water that would result from the condensation of all the water in a column of atmosphere. A PWV of $1 \mathrm{~mm}$ corresponds to a column density of $1 \mathrm{~kg} \mathrm{~m}^{-2}$. Espy and Hammond (1995) consider PWVs of $29,21,8.5$, and $4.2 \mathrm{~mm}$, corresponding respectively to low-latitude summer, winter, high-latitude summer, and winter.

\subsection{Determining hydroxyl temperatures}

If we assume a Boltzmann distribution for the population of rotational levels, the intensity $I$ of a Meinel band line is (Mies, 1974)

$I=N_{v^{\prime}} A \frac{2\left(2 J^{\prime}+1\right)}{Q_{v^{\prime}}(T)} \exp \left[\frac{-h c F}{k T}\right]$

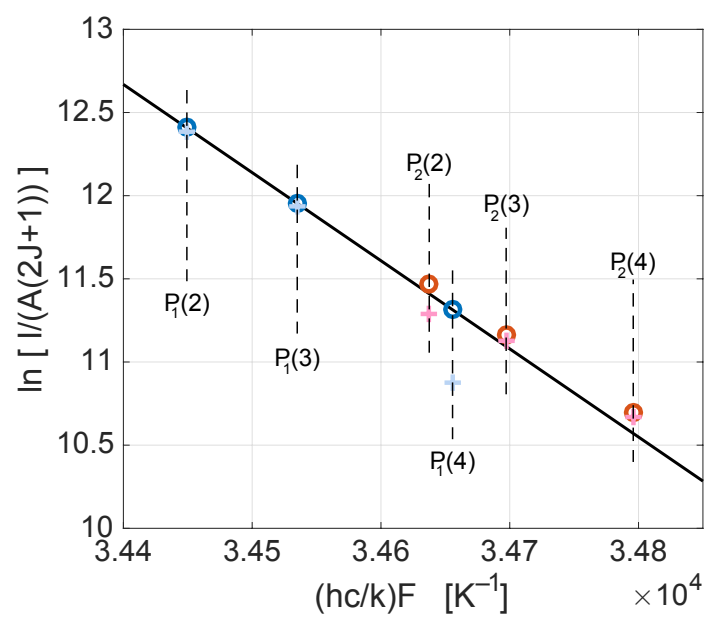

Figure 3. Boltzmann plot determined from the $\mathrm{OH}(8-3)$ P-branch lines shown in the HiTIES spectrum from Fig. 2, taken on 23 December 2003 at 22:30 UT, with an integration time of $120 \mathrm{~s}$. Blue symbols represent $\mathrm{P}_{1}$ lines, and red symbols are $\mathrm{P}_{2}$ lines. Crosses indicate intensities as measured by the HiTIES instrument, whereas open circles have been corrected for water vapour absorption. The solid black line is a linear fit to the water-vapour-corrected $\mathrm{P}_{1}$ lines. The slope of this fit gives a rotational temperature of $T=188.7 \mathrm{~K}$.

where $N_{v^{\prime}}$ is the total number of molecules in the $v^{\prime}$ vibrational level, $A$ is the transition probability, $J^{\prime}$ is the upper state total angular momentum quantum number, $Q_{v^{\prime}}$ is the partition function, $F$ is the energy level of the initial rotational level, $T$ is the rotational temperature, $h$ is Planck's constant, $c$ is the speed of light, and $k$ is Boltzmann's constant.

One method to obtain the temperature is to take the ratio of intensities of $\mathrm{OH}$ lines with different upper rotational states (Phillips et al., 2004). Thus, from Eq. (3), we obtain

$T=\frac{h c\left(F_{b}-F_{a}\right)}{k}\left\{\ln \left[\frac{I_{a} A_{b}\left(2 J_{b}^{\prime}+1\right)}{I_{b} A_{a}\left(2 J_{a}^{\prime}+1\right)}\right]\right\}^{-1}$,

where the indices $a$ and $b$ represent the two emission lines from different upper states.

Values for the energy levels are taken from Coxon and Foster (1982). There is a discrepancy in the literature over transition probabilities that can affect the temperature values obtained from Eq. (4) by up to $\sim 10-20 \mathrm{~K}$ (Phillips et al., 2004; Perminov et al., 2007). The most common sources are Mies (1974), Langhoff et al. (1986), and Turnbull and Lowe (1989). It is therefore important to be consistent with the set of transition probabilities used whenever comparing temperature results. In the results shown in this paper, we use the values from Mies (1974).

Figure 2 shows a clear-sky spectrum taken with the HiTIES instrument. Only $\mathrm{OH}$ airglow emission has been recorded; there are no auroral emissions which, when present, can contaminate the $\mathrm{OH}$ lines, rendering the measurement of $\mathrm{OH}$ line intensities more difficult. We have de- 
Table 1. Water vapour transmission at wavelengths of $\mathrm{OH}(8-3)$ lines. Vacuum wavelengths are from Rousselot et al. (2000), with conversion to air wavelengths undertaken as specified by Ciddor (1996). At each wavelength, water vapour transmission is given by a function of precipitable water vapour (PWV): $T_{\lambda}=\exp (-A \times$ PWV), with PWV in mm.

\begin{tabular}{lrlr}
\hline $\begin{array}{r}\text { Wavelength } \\
\text { vacuum }(\AA)\end{array}$ & $\begin{array}{r}\text { Wavelength } \\
\text { air }(\AA)\end{array}$ & Transition & $\begin{array}{r}A \\
\left(\mathrm{~mm}^{-1}\right)\end{array}$ \\
\hline 7277.100 & 7275.095 & $\mathrm{Q}_{2}(1) \mathrm{e}$ & $4.36 \times 10^{-3}$ \\
7277.125 & 7275.120 & $\mathrm{Q}_{2}(1) \mathrm{f}$ & $5.04 \times 10^{-3}$ \\
7278.405 & 7276.400 & $\mathrm{Q}_{1}(1) \mathrm{e}$ & $1.57 \times 10^{-2}$ \\
7278.415 & 7276.410 & $\mathrm{Q}_{1}(1) \mathrm{f}$ & $1.44 \times 10^{-2}$ \\
7286.428 & 7284.421 & $\mathrm{Q}_{1}(2) \mathrm{e}$ & $2.18 \times 10^{-4}$ \\
7286.464 & 7284.457 & $\mathrm{Q}_{1}(2) \mathrm{f}$ & $1.92 \times 10^{-4}$ \\
7297.911 & 7295.901 & $\mathrm{Q}_{1}(3) \mathrm{e}$ & $9.31 \times 10^{-4}$ \\
7297.988 & 7295.978 & $\mathrm{Q}_{1}(3) \mathrm{f}$ & $7.15 \times 10^{-4}$ \\
7305.691 & 7303.679 & $\mathrm{P}_{2}(2) \mathrm{e}$ & $5.92 \times 10^{-3}$ \\
7305.766 & 7303.754 & $\mathrm{P}_{2}(2) \mathrm{f}$ & $5.69 \times 10^{-3}$ \\
7318.262 & 7316.246 & $\mathrm{P}_{1}(2) \mathrm{e}$ & $7.24 \times 10^{-4}$ \\
7318.334 & 7316.318 & $\mathrm{P}_{1}(2) \mathrm{f}$ & $7.22 \times 10^{-4}$ \\
7331.142 & 7329.123 & $\mathrm{P}_{2}(3) \mathrm{e}$ & $7.51 \times 10^{-4}$ \\
7331.192 & 7329.173 & $\mathrm{P}_{2}(3) \mathrm{f}$ & $1.89 \times 10^{-3}$ \\
7342.835 & 7340.813 & $\mathrm{P}_{1}(3) \mathrm{e}$ & $8.42 \times 10^{-4}$ \\
7342.979 & 7340.956 & $\mathrm{P}_{1}(3) \mathrm{f}$ & $3.23 \times 10^{-4}$ \\
7360.684 & 7358.657 & $\mathrm{P}_{2}(4) \mathrm{e}$ & $7.74 \times 10^{-4}$ \\
7360.688 & 7358.661 & $\mathrm{P}_{2}(4) \mathrm{f}$ & $8.01 \times 10^{-4}$ \\
7371.278 & 7369.248 & $\mathrm{P}_{1}(4) \mathrm{e}$ & $2.55 \times 10^{-2}$ \\
7371.513 & 7369.483 & $\mathrm{P}_{1}(4) \mathrm{f}$ & $2.62 \times 10^{-3}$ \\
7394.205 & 7392.169 & $\mathrm{P}_{2}(5) \mathrm{e}$ & $1.60 \times 10^{-4}$ \\
7394.264 & 7392.228 & $\mathrm{P}_{2}(5) \mathrm{f}$ & $1.69 \times 10^{-4}$ \\
7403.727 & 7401.688 & $\mathrm{P}_{1}(5) \mathrm{e}$ & $6.09 \times 10^{-5}$ \\
7404.067 & 7402.028 & $\mathrm{P}_{1}(5) \mathrm{f}$ & $6.98 \times 10^{-5}$ \\
\hline & & & \\
\hline & & & \\
730 & & &
\end{tabular}

veloped a fitting routine with which we can determine the intensities of $\mathrm{OH}$ lines, even when there is auroral contamination. A least squares fit is used, involving four different components present in the measured spectrum: a constant background, six $\mathrm{OH}$ emission lines, two auroral $\mathrm{O}^{+}$doublets (at wavelengths $\lambda=7320.121 \AA$ and $7319.044 \AA$ and $\lambda=7330.755 \AA$ and $7329.675 \AA$; from Sharpee et al., 2004), and auroral $\mathrm{N}_{2} 1 \mathrm{P}(5-3)$ band emission, which can be modelled (Whiter et al., 2014).

For the spectrum in Fig. 2, the $\mathrm{OH}$ line intensities can be easily determined as there is no auroral contamination. We determine temperatures using the three strongest lines: $\mathrm{P}_{1}(2), \mathrm{P}_{1}(3)$, and $\mathrm{P}_{1}(4)$. Since there are three possible line ratios using these lines, we obtain three temperature values. Applying Eq. (4), we obtain $T\left(\mathrm{P}_{1}(2) / \mathrm{P}_{1}(3)\right)=189.1 \mathrm{~K}$, $T\left(\mathrm{P}_{1}(3) / \mathrm{P}_{1}(4)\right)=183.7 \mathrm{~K}$, and $T\left(\mathrm{P}_{1}(3) / \mathrm{P}_{1}(4)\right)=180.0 \mathrm{~K}$. The difference between these three values is due to water vapour absorption that affects the $\mathrm{P}_{1}(4)$ lines, whereas $\mathrm{P}_{1}(2)$ and $\mathrm{P}_{1}(3)$ are much less affected. Correcting for water vapour absorption using the modelling described in Sect. 2.1,
Table 2. Water vapour transmission at wavelengths of $\mathrm{OH}(5-1)$ lines. Vacuum wavelengths are from Rousselot et al. (2000), with conversion to air wavelengths undertaken as specified by Ciddor (1996). At each wavelength, water vapour transmission is given by a function of PWV: $T_{\lambda}=\exp (-A \times \mathrm{PWV})$, with PWV in $\mathrm{mm}$.

\begin{tabular}{lrlr}
\hline $\begin{array}{l}\text { Wavelength } \\
\text { vacuum }(\AA)\end{array}$ & $\begin{array}{r}\text { Wavelength } \\
\text { air }(\AA)\end{array}$ & Transition & $\begin{array}{r}A \\
\left(\mathrm{~mm}^{-1}\right)\end{array}$ \\
\hline 7914.418 & 7912.241 & $\mathrm{Q}_{2}(1) \mathrm{e}$ & $3.24 \times 10^{-4}$ \\
7914.438 & 7912.261 & $\mathrm{Q}_{2}(1) \mathrm{f}$ & $2.79 \times 10^{-4}$ \\
7915.880 & 7913.703 & $\mathrm{Q}_{1}(1) \mathrm{e}$ & $2.40 \times 10^{-5}$ \\
7915.890 & 7913.713 & $\mathrm{Q}_{1}(1) \mathrm{f}$ & $2.41 \times 10^{-5}$ \\
7920.513 & 7918.335 & $\mathrm{Q}_{2}(2) \mathrm{e}$ & $1.43 \times 10^{-4}$ \\
7920.542 & 7918.364 & $\mathrm{Q}_{2}(2) \mathrm{f}$ & $1.35 \times 10^{-4}$ \\
7923.281 & 7921.102 & $\mathrm{Q}_{1}(2) \mathrm{e}$ & $7.28 \times 10^{-4}$ \\
7923.315 & 7921.136 & $\mathrm{Q}_{1}(2) \mathrm{f}$ & $6.42 \times 10^{-4}$ \\
7933.913 & 7931.731 & $\mathrm{Q}_{1}(3) \mathrm{e}$ & $6.84 \times 10^{-3}$ \\
7933.986 & 7931.804 & $\mathrm{Q}_{1}(3) \mathrm{f}$ & $7.51 \times 10^{-3}$ \\
7951.349 & 7949.162 & $\mathrm{P}_{2}(2) \mathrm{e}$ & $3.63 \times 10^{-5}$ \\
7951.432 & 7949.245 & $\mathrm{P}_{2}(2) \mathrm{f}$ & $3.56 \times 10^{-5}$ \\
7966.793 & 7964.602 & $\mathrm{P}_{1}(2) \mathrm{e}$ & $8.74 \times 10^{-4}$ \\
7966.889 & 7964.698 & $\mathrm{P}_{1}(2) \mathrm{f}$ & $5.40 \times 10^{-4}$ \\
7981.943 & 7979.748 & $\mathrm{P}_{2}(3) \mathrm{e}$ & $3.06 \times 10^{-4}$ \\
7981.983 & 7979.788 & $\mathrm{P}_{2}(3) \mathrm{f}$ & $4.04 \times 10^{-4}$ \\
7995.439 & 7993.240 & $\mathrm{P}_{1}(3) \mathrm{e}$ & $3.26 \times 10^{-4}$ \\
7995.622 & 7993.423 & $\mathrm{P}_{1}(3) \mathrm{f}$ & $1.03 \times 10^{-3}$ \\
8016.250 & 8014.046 & $\mathrm{P}_{2}(4) \mathrm{e}$ & $1.02 \times 10^{-2}$ \\
8016.276 & 8014.072 & $\mathrm{P}_{2}(4) \mathrm{f}$ & $7.70 \times 10^{-3}$ \\
8027.875 & 8025.668 & $\mathrm{P}_{1}(4) \mathrm{e}$ & $2.89 \times 10^{-4}$ \\
8028.160 & 8025.953 & $\mathrm{P}_{1}(4) \mathrm{f}$ & $1.16 \times 10^{-3}$ \\
8054.180 & 8051.966 & $\mathrm{P}_{2}(5) \mathrm{f}$ & $1.48 \times 10^{-4}$ \\
8054.289 & 8052.075 & $\mathrm{P}_{2}(5) \mathrm{e}$ & $2.21 \times 10^{-4}$ \\
8064.196 & 8061.979 & $\mathrm{P}_{1}(5) \mathrm{e}$ & $4.40 \times 10^{-4}$ \\
8064.594 & 8062.377 & $\mathrm{P}_{1}(5) \mathrm{f}$ & $4.19 \times 10^{-4}$ \\
\hline & & &
\end{tabular}

we obtain very similar values from all three line ratios: $T\left(\mathrm{P}_{1}(2) / \mathrm{P}_{1}(3)\right)=188.7 \mathrm{~K}, T\left(\mathrm{P}_{1}(3) / \mathrm{P}_{1}(4)\right)=188.6 \mathrm{~K}$, and $T\left(\mathrm{P}_{1}(3) / \mathrm{P}_{1}(4)\right)=188.5 \mathrm{~K}$.

In order to ensure that the airglow layer is in LTE, we produce a Boltzmann plot (e.g. Sigernes et al., 2003) for each temperature retrieval performed. This consists of plotting $\ln \{I /[A(2 J+1)]\}$ as a function of $(h c / k) F$ for each of the $\mathrm{OH}(8-3) \mathrm{P}$-branch lines measured by HiTIES. If Eq. (3) is valid, i.e. the airglow layer is in LTE, it can be seen that the plotted function should be linear and the slope is the rotational temperature $T$. As an example, the Boltzmann plot for the HiTIES spectrum in Fig. 2 (measured on 23 December 2003 at 22:30 UT) is shown in Fig. 3. The linear fit (solid black line) is determined using the three $\mathrm{P}_{1}$ lines (blue symbols), since these have the highest intensities (and thus lower associated errors). The points corresponding to the $\mathrm{P}_{2}$ lines (represented by red symbols in Fig. 3) follow the same linear fit, indicating that the airglow layer is indeed in LTE at this time. To assert that there is LTE, we use the same criteria as 
Table 3. Water vapour transmission at wavelengths of $\mathrm{OH}(9-4)$ lines. Vacuum wavelengths are from Rousselot et al. (2000), with conversion to air wavelengths undertaken as specified by Ciddor (1996). At each wavelength, water vapour transmission is given by a function of PWV: $T_{\lambda}=\exp (-A \times \mathrm{PWV})$, with PWV in $\mathrm{mm}$.

\begin{tabular}{|c|c|c|c|}
\hline $\begin{array}{l}\text { Wavelength } \\
\operatorname{vacuum}(\AA)\end{array}$ & $\begin{array}{r}\text { Wavelength } \\
\operatorname{air}(\AA)\end{array}$ & Transition & $\begin{array}{r}A \\
\left(\mathrm{~mm}^{-1}\right)\end{array}$ \\
\hline 7751.541 & 7749.408 & $\mathrm{Q}_{2}(1) \mathrm{f}$ & $1.15 \times 10^{-8}$ \\
\hline 7751.574 & 7749.441 & $\mathrm{Q}_{2}(1) \mathrm{e}$ & $8.23 \times 10^{-9}$ \\
\hline 7752.768 & 7750.635 & $\mathrm{Q}_{1}(1) \mathrm{f}$ & $3.77 \times 10^{-8}$ \\
\hline 7752.779 & 7750.646 & $\mathrm{Q}_{1}(1) \mathrm{e}$ & $3.89 \times 10^{-8}$ \\
\hline 7758.967 & 7756.832 & $\mathrm{Q}_{2}(2) \mathrm{f}$ & $3.84 \times 10^{-7}$ \\
\hline 7759.021 & 7756.886 & $\mathrm{Q}_{2}(2) \mathrm{e}$ & $3.64 \times 10^{-7}$ \\
\hline 7762.111 & 7759.975 & $\mathrm{Q}_{1}(2) \mathrm{f}$ & $4.85 \times 10^{-6}$ \\
\hline 7762.152 & 7760.016 & $\mathrm{Q}_{1}(2) \mathrm{e}$ & $3.11 \times 10^{-6}$ \\
\hline 7775.465 & 7773.326 & $\mathrm{Q}_{1}(3) \mathrm{f}$ & $1.16 \times 10^{-6}$ \\
\hline 7775.555 & 7773.416 & $\mathrm{Q}_{1}(3) \mathrm{e}$ & $4.31 \times 10^{-6}$ \\
\hline 7782.529 & 7780.388 & $\mathrm{P}_{2}(2) \mathrm{e}$ & $4.58 \times 10^{-8}$ \\
\hline 7782.616 & 7780.475 & $\mathrm{P}_{2}(2) \mathrm{f}$ & $4.90 \times 10^{-8}$ \\
\hline 7793.001 & 7790.857 & $\mathrm{Q}_{1}(4) \mathrm{f}$ & $1.86 \times 10^{-6}$ \\
\hline 7793.159 & 7791.015 & $\mathrm{Q}_{1}(4) \mathrm{e}$ & $2.18 \times 10^{-6}$ \\
\hline 7796.218 & 7794.073 & $\mathrm{P}_{1}(2) \mathrm{e}$ & $9.22 \times 10^{-7}$ \\
\hline 7796.294 & 7794.149 & $\mathrm{P}_{1}(2) \mathrm{f}$ & $1.05 \times 10^{-6}$ \\
\hline 7810.581 & 7808.432 & $\mathrm{P}_{2}(3) \mathrm{e}$ & $2.54 \times 10^{-6}$ \\
\hline 7810.650 & 7808.501 & $\mathrm{P}_{2}(3) \mathrm{f}$ & $5.92 \times 10^{-6}$ \\
\hline 7823.578 & 7821.426 & $\mathrm{P}_{1}(3) \mathrm{e}$ & $3.19 \times 10^{-6}$ \\
\hline 7823.732 & 7821.580 & $\mathrm{P}_{1}(3) \mathrm{f}$ & $3.12 \times 10^{-6}$ \\
\hline 7843.411 & 7841.254 & $\mathrm{P}_{2}(4) \mathrm{e}$ & $1.39 \times 10^{-5}$ \\
\hline 7843.436 & 7841.278 & $\mathrm{P}_{2}(4) \mathrm{f}$ & $1.19 \times 10^{-5}$ \\
\hline 7855.393 & 7853.232 & $\mathrm{P}_{1}(4) \mathrm{e}$ & $2.44 \times 10^{-5}$ \\
\hline 7855.648 & 7853.487 & $P_{1}(4) f$ & $3.85 \times 10^{-4}$ \\
\hline 7880.929 & 7878.761 & $\mathrm{P}_{2}(5) \mathrm{f}$ & $4.58 \times 10^{-5}$ \\
\hline 7880.971 & 7878.803 & $\mathrm{P}_{2}(5) \mathrm{e}$ & $1.17 \times 10^{-4}$ \\
\hline 7891.834 & 7889.663 & $\mathrm{P}_{1}(5) \mathrm{e}$ & $2.53 \times 10^{-4}$ \\
\hline 7892.209 & 7890.039 & $P_{1}(5) f$ & $1.43 \times 10^{-4}$ \\
\hline 7923.161 & 7920.982 & $\mathrm{P}_{2}(6) \mathrm{e}$ & $1.36 \times 10^{-3}$ \\
\hline 7923.286 & 7921.107 & $\mathrm{P}_{2}(6) \mathrm{f}$ & $7.13 \times 10^{-4}$ \\
\hline 7933.049 & 7930.867 & $\mathrm{P}_{1}(6) \mathrm{e}$ & $9.01 \times 10^{-5}$ \\
\hline 7933.561 & 7931.379 & $P_{1}(6) f$ & $2.30 \times 10^{-4}$ \\
\hline 7970.216 & 7968.024 & $\mathrm{P}_{2}(7) \mathrm{f}$ & $5.12 \times 10^{-3}$ \\
\hline 7970.440 & 7968.248 & $\mathrm{P}_{2}(7) \mathrm{e}$ & $2.48 \times 10^{-3}$ \\
\hline 7979.181 & 7976.987 & $\mathrm{P}_{1}(7) \mathrm{e}$ & $1.38 \times 10^{-4}$ \\
\hline 7979.844 & 7977.650 & $\mathrm{P}_{1}(7) \mathrm{f}$ & $1.17 \times 10^{-4}$ \\
\hline
\end{tabular}

Sigernes et al. (2003), namely a variance between the fit and the $P_{1}$ values of less than 0.05 and between the fit and the $P_{2}$ values of 0.3 .

The effect of taking into account water vapour absorption in the $\mathrm{OH}$ line intensities is also shown in the Boltzmann plot of Fig. 3. Circles are used to represent water vapourcorrected $\mathrm{OH}$ intensities, whereas crosses represent uncorrected intensities. From this figure it can be seen that the lines most absorbed by water vapour are $\mathrm{P}_{1}(4)$ and $\mathrm{P}_{2}(2)$. Without
Table 4. Water vapour transmission at wavelengths of $\mathrm{OH}(6-2)$ lines. Vacuum wavelengths are from Rousselot et al. (2000), with conversion to air wavelengths undertaken as specified by Ciddor (1996). At each wavelength, water vapour transmission is given by a function of PWV: $T_{\lambda}=\exp (-A \times \mathrm{PWV})$, with PWV in $\mathrm{mm}$.

\begin{tabular}{|c|c|c|c|}
\hline $\begin{array}{l}\text { Wavelength } \\
\text { vacuum }(\AA)\end{array}$ & $\begin{array}{r}\text { Wavelength } \\
\operatorname{air}(\AA)\end{array}$ & Transition & $\begin{array}{r}A \\
\left(\mathrm{~mm}^{-1}\right)\end{array}$ \\
\hline 8345.315 & 8343.022 & $\mathrm{Q}_{2}(1) \mathrm{f}$ & $2.25 \times 10^{-4}$ \\
\hline 8345.337 & 8343.044 & $\mathrm{Q}_{2}(1) \mathrm{e}$ & $2.20 \times 10^{-4}$ \\
\hline 8346.891 & 8344.598 & $\mathrm{Q}_{1}(1) \mathrm{f}$ & $2.69 \times 10^{-4}$ \\
\hline 8346.901 & 8344.608 & $\mathrm{Q}_{1}(1) \mathrm{e}$ & $2.94 \times 10^{-4}$ \\
\hline 8352.051 & 8349.756 & $\mathrm{Q}_{2}(2) \mathrm{f}$ & $7.71 \times 10^{-4}$ \\
\hline 8352.084 & 8349.789 & $\mathrm{Q}_{2}(2) \mathrm{e}$ & $7.53 \times 10^{-4}$ \\
\hline 8355.143 & 8352.847 & $\mathrm{Q}_{1}(2) \mathrm{f}$ & $4.10 \times 10^{-3}$ \\
\hline 8355.181 & 8352.885 & $\mathrm{Q}_{1}(2) \mathrm{e}$ & $2.27 \times 10^{-3}$ \\
\hline 8366.979 & 8364.680 & $\mathrm{Q}_{1}(3) \mathrm{f}$ & $5.39 \times 10^{-4}$ \\
\hline 8367.060 & 8364.761 & $\mathrm{Q}_{1}(3) \mathrm{e}$ & $4.62 \times 10^{-4}$ \\
\hline 8382.554 & 8380.251 & $\mathrm{Q}_{1}(4) \mathrm{f}$ & $9.80 \times 10^{-5}$ \\
\hline 8382.692 & 8380.389 & $\mathrm{Q}_{1}(4) \mathrm{e}$ & $1.48 \times 10^{-4}$ \\
\hline 8384.650 & 8382.346 & $\mathrm{P}_{2}(2) \mathrm{e}$ & $3.26 \times 10^{-4}$ \\
\hline 8384.740 & 8382.436 & $\mathrm{P}_{2}(2) \mathrm{f}$ & $1.45 \times 10^{-4}$ \\
\hline 8401.429 & 8399.121 & $\mathrm{P}_{1}(2) \mathrm{e}$ & $4.61 \times 10^{-5}$ \\
\hline 8401.527 & 8399.219 & $P_{1}(2) f$ & $7.56 \times 10^{-5}$ \\
\hline 8417.520 & 8415.207 & $\mathrm{P}_{2}(3) \mathrm{e}$ & $3.19 \times 10^{-3}$ \\
\hline 8417.566 & 8415.253 & $\mathrm{P}_{2}(3) \mathrm{f}$ & $3.79 \times 10^{-3}$ \\
\hline 8432.396 & 8430.079 & $\mathrm{P}_{1}(3) \mathrm{e}$ & $6.94 \times 10^{-4}$ \\
\hline 8432.586 & 8430.269 & $P_{1}(3) f$ & $2.81 \times 10^{-4}$ \\
\hline 8454.562 & 8452.239 & $\mathrm{P}_{2}(4) \mathrm{f}$ & $5.69 \times 10^{-4}$ \\
\hline 8454.583 & 8452.260 & $\mathrm{P}_{2}(4) \mathrm{e}$ & $4.56 \times 10^{-4}$ \\
\hline 8467.534 & 8465.208 & $\mathrm{P}_{1}(4) \mathrm{e}$ & $2.28 \times 10^{-5}$ \\
\hline 8467.835 & 8465.509 & $P_{1}(4) f$ & $3.65 \times 10^{-6}$ \\
\hline 8495.669 & 8493.335 & $P_{2}(5) f$ & $8.26 \times 10^{-5}$ \\
\hline 8495.776 & 8493.442 & $\mathrm{P}_{2}(5) \mathrm{e}$ & $1.33 \times 10^{-4}$ \\
\hline 8506.965 & 8504.628 & $\mathrm{P}_{1}(5) \mathrm{e}$ & $2.51 \times 10^{-5}$ \\
\hline 8507.391 & 8505.054 & $P_{1}(5) f$ & $4.26 \times 10^{-5}$ \\
\hline 8540.919 & 8538.573 & $P_{2}(6) f$ & $1.78 \times 10^{-4}$ \\
\hline 8541.126 & 8538.780 & $\mathrm{P}_{2}(6) \mathrm{e}$ & $6.82 \times 10^{-5}$ \\
\hline 8550.776 & 8548.427 & $P_{1}(6) \mathrm{e}$ & $1.61 \times 10^{-5}$ \\
\hline 8551.337 & 8548.988 & $P_{1}(6) f$ & $1.78 \times 10^{-5}$ \\
\hline 8590.396 & 8588.037 & $P_{2}(7) f$ & $5.10 \times 10^{-6}$ \\
\hline 8590.713 & 8588.354 & $\mathrm{P}_{2}(7) \mathrm{e}$ & $9.94 \times 10^{-6}$ \\
\hline 8599.047 & 8596.685 & $\mathrm{P}_{1}(7) \mathrm{e}$ & $3.02 \times 10^{-5}$ \\
\hline 8599.755 & 8597.393 & $P_{1}(7) f$ & $2.74 \times 10^{-5}$ \\
\hline
\end{tabular}

correcting the hydroxyl emission line intensities for this effect, we would obtain a bad linear fit and a significant error on the rotational temperature.

In the following section, Sect. 3, we detail the results of water vapour absorption modelling, providing coefficients for scaling $\mathrm{OH}$ line intensities to take this into account. More details on the resulting temperatures determined from $\mathrm{Hi}-$ TIES measurements are shown in Sect. 4. 
Table 5. Water vapour transmission at wavelengths of $\mathrm{OH}(8-4)$ lines. Vacuum wavelengths are from Rousselot et al. (2000), with conversion to air wavelengths undertaken as specified by Ciddor (1996). At each wavelength, water vapour transmission is given by a function of PWV: $T_{\lambda}=\exp (-A \times \mathrm{PWV})$, with $\mathrm{PWV}$ in $\mathrm{mm}$.

\begin{tabular}{lrlr}
\hline Wavelength & Wavelength & Transition & $\begin{array}{c}A \\
\text { vacuum }(\AA)\end{array}$ \\
air $(\AA)$ & & $5.59 \times 10^{-2}$ \\
\hline 9376.874 & 9374.302 & $\mathrm{Q}_{2}(1) \mathrm{f}$ & $4.21 \times 10^{-2}$ \\
9376.907 & 9374.335 & $\mathrm{Q}_{2}(1) \mathrm{e}$ & $2.07 \times 10^{-2}$ \\
9378.526 & 9375.953 & $\mathrm{Q}_{1}(1) \mathrm{f}$ & $2.07 \times 10^{-2}$ \\
9378.540 & 9375.967 & $\mathrm{Q}_{1}(1) \mathrm{e}$ & $2.35 \times 10^{-2}$ \\
9385.458 & 9382.884 & $\mathrm{Q}_{2}(2) \mathrm{f}$ & 2.35 \\
9385.513 & 9382.939 & $\mathrm{Q}_{2}(2) \mathrm{e}$ & $2.24 \times 10^{-2}$ \\
9389.260 & 9386.685 & $\mathrm{Q}_{1}(2) \mathrm{f}$ & 2.41 \\
9389.307 & 9386.732 & $\mathrm{Q}_{1}(2) \mathrm{e}$ & 1.65 \\
9404.609 & 9402.029 & $\mathrm{Q}_{1}(3) \mathrm{e}$ & $2.43 \times 10^{-2}$ \\
9404.713 & 9402.133 & $\mathrm{Q}_{1}(3) \mathrm{f}$ & $1.84 \times 10^{-2}$ \\
9422.257 & 9419.673 & $\mathrm{P}_{2}(2) \mathrm{e}$ & $9.19 \times 10^{-3}$ \\
9422.370 & 9419.786 & $\mathrm{P}_{2}(2) \mathrm{f}$ & $7.87 \times 10^{-3}$ \\
9442.186 & 9439.596 & $\mathrm{P}_{1}(2) \mathrm{e}$ & $5.62 \times 10^{-2}$ \\
9442.294 & 9439.704 & $\mathrm{P}_{1}(2) \mathrm{f}$ & $6.53 \times 10^{-2}$ \\
9461.085 & 9458.490 & $\mathrm{P}_{2}(3) \mathrm{f}$ & $2.07 \times 10^{-2}$ \\
9461.161 & 9458.566 & $\mathrm{P}_{2}(3) \mathrm{e}$ & $2.35 \times 10^{-2}$ \\
9479.348 & 9476.748 & $\mathrm{P}_{1}(3) \mathrm{e}$ & $1.19 \times 10^{-1}$ \\
9479.561 & 9476.961 & $\mathrm{P}_{1}(3) \mathrm{f}$ & $1.69 \times 10^{-2}$ \\
9505.418 & 9502.811 & $\mathrm{P}_{2}(4) \mathrm{f}$ & $3.93 \times 10^{-2}$ \\
9505.425 & 9502.818 & $\mathrm{P}_{2}(4) \mathrm{e}$ & $3.97 \times 10^{-2}$ \\
9521.793 & 9519.182 & $\mathrm{P}_{1}(4) \mathrm{e}$ & $2.60 \times 10^{-1}$ \\
9522.139 & 9519.528 & $\mathrm{P}_{1}(4) \mathrm{f}$ & $2.01 \times 10^{-1}$ \\
9555.110 & 9552.490 & $\mathrm{P}_{2}(5) \mathrm{f}$ & $2.19 \times 10^{-2}$ \\
9555.199 & 9552.579 & $\mathrm{P}_{2}(5) \mathrm{e}$ & $2.12 \times 10^{-2}$ \\
9569.714 & 9567.090 & $\mathrm{P}_{1}(5) \mathrm{e}$ & $4.63 \times 10^{-2}$ \\
9570.213 & 9567.589 & $\mathrm{P}_{1}(5) \mathrm{f}$ & $1.21 \times 10^{-2}$ \\
9610.261 & 9607.626 & $\mathrm{P}_{2}(6) \mathrm{f}$ & $9.91 \times 10^{-3}$ \\
9610.463 & 9607.828 & $\mathrm{P}_{2}(6) \mathrm{e}$ & $5.11 \times 10^{-2}$ \\
9623.270 & 9620.631 & $\mathrm{P}_{1}(6) \mathrm{e}$ & $2.62 \times 10^{-2}$ \\
9623.938 & 9621.299 & $\mathrm{P}_{1}(6) \mathrm{f}$ & $5.28 \times 10^{-1}$ \\
9670.988 & 9668.336 & $\mathrm{P}_{2}(7) \mathrm{f}$ & $1.16 \times 10^{-2}$ \\
9671.319 & 9668.667 & $\mathrm{P}_{2}(7) \mathrm{e}$ & $3.07 \times 10^{-3}$ \\
9682.607 & 9679.952 & $\mathrm{P}_{1}(7) \mathrm{e}$ & $2.84 \times 10^{-2}$ \\
9683.466 & 9680.811 & $\mathrm{P}_{1}(7) \mathrm{f}$ & $3.16 \times 10^{-2}$ \\
\hline & & &
\end{tabular}

\section{Water vapour transmission}

We have determined water vapour transmission for hydroxyl emissions for the strongest transitions in the (8-3) band (Table 1), the (5-1) band (Table 2), the (9-4) band (Table 3), the (6-2) band (Table 4), and the (8-4) band (Table 5).

Water vapour absorption lines originating in the troposphere are mainly subject to pressure-broadening. Using the HITRAN model, we find that for a temperature of about $300 \mathrm{~K}$ and a pressure of $1 \mathrm{bar}$ (most $\mathrm{H}_{2} \mathrm{O}$ is concentrated near the Earth's surface), the full width at half maximum of an
$\mathrm{H}_{2} \mathrm{O}$ line at a wavelength close to the $\mathrm{OH}(8-3) \mathrm{P}_{1}(4)$ line is about $0.1 \AA$. On the other hand, since $\mathrm{OH}$ emission is produced near the mesopause, the principal broadening mechanism is Doppler broadening. The full width at half maximum of such a Gaussian line profile is given by

$\Delta \lambda=2 \lambda_{0} \sqrt{2 \ln (2) \frac{k T}{m c^{2}}}$,

where $\lambda_{0}$ is the centre wavelength of the line. Applying Eq. (5) using the value of temperature determined in Sect. 2.2 from the HiTIES spectrum in Fig. 2, $T=188.7 \mathrm{~K}$, gives $\Delta \lambda / \lambda_{0}=2.38 \times 10^{-6}$. Therefore, at the wavelength of the most absorbed $\mathrm{OH}$ line in the HiTIES spectrum, $\mathrm{P}_{1}(4)$, we obtain a full width at half maximum of $0.018 \AA$. We consider that this is sufficiently small compared to the width of the water vapour absorption lines that we do not calculate the precise shape of the $\mathrm{OH}$ lines to determine the $\mathrm{H}_{2} \mathrm{O}$ transmission values in Tables $1-5$, instead only calculating the values at the line centre wavelength.

In addition, we do not take into account any Doppler shifts that may occur to the centre wavelengths, or effects of nonvertical lines of sight. Thus, the values given are only valid when observing in the zenith, assuming that any vertical flows are small.

The water vapour transmissions of $\mathrm{OH}(8-3)$ band emission lines, some of which are observed by the HiTIES instrument (see Fig. 2), are plotted in Fig. 4. The two lambdadoubled components of each line are shown, even though $\mathrm{Hi}$ TIES does not have sufficiently high wavelength resolution to be able to distinguish these. Of the lines observed by HiTIES, it is mainly $\mathrm{P}_{1}(4) e$ that is affected. For the case of the HiTIES observation from 23 December 2003 at 22:30 UT, described in Sect. 2.2, a value of PWV of $2.7 \mathrm{~mm}$ was taken in order for the three hydroxyl temperatures (obtained from ratios $\mathrm{P}_{1}(2) / \mathrm{P}_{1}(3), \mathrm{P}_{1}(2) / \mathrm{P}_{1}(4)$, and $\mathrm{P}_{1}(3) / \mathrm{P}_{1}(4)$ ) to match. This represents an absorption of $6.7 \%$ of the $\mathrm{OH}(8$ 3) $\mathrm{P}_{1}(4) \mathrm{e}$ line.

Plots of transmission as a function of PWV for lines from the $\mathrm{OH}$ bands (5-1), (9-4), (6-2), and (8-4) are provided in Figs. 5-8. These plots show that the (8-4) band is particularly affected by water vapour absorption.

In this study, we focus on the role of water vapour absorption; however, other species may also participate in the attenuation of hydroxyl line emission. As a test, we estimate the absorption by $\mathrm{CO}_{2}$ of $\mathrm{OH}$ line emission using a similar method to that used for $\mathrm{H}_{2} \mathrm{O}$ absorption described in Sect. 2.1. We use a profile of $\mathrm{CO}_{2}$ density with altitude from Foucher et al. (2011), scaled to the high-latitude winter carbon dioxide column density given by Espy and Hammond (1995) of $7.103 \times 10^{21}$ molecules $\mathrm{cm}^{-2}$. The HITRAN database is then used to determine $\mathrm{CO}_{2}$ absorption cross sections. We find that the transmission coefficients at the centre wavelengths of all the $\mathrm{OH}$ lines included in Tables 1 to 5 are equal to 1 . Thus, at the hydroxyl line wavelengths included 


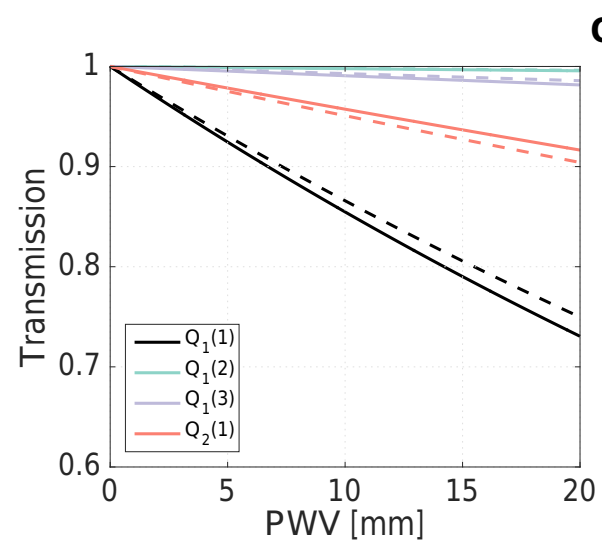

OH(8-3)

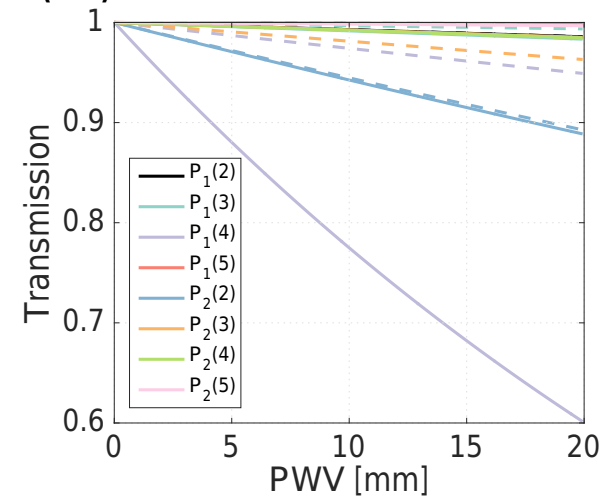

Figure 4. Water vapour transmission of the $\mathrm{OH}(8-3)$ lines, measured by HiTIES, as a function of precipitable water vapour (PWV). The left panel shows the Q-branch lines and the right panel, the P branch. Solid lines represent the e component of the lambda-doubled line, and the dashed lines represent the $\mathrm{f}$ component.

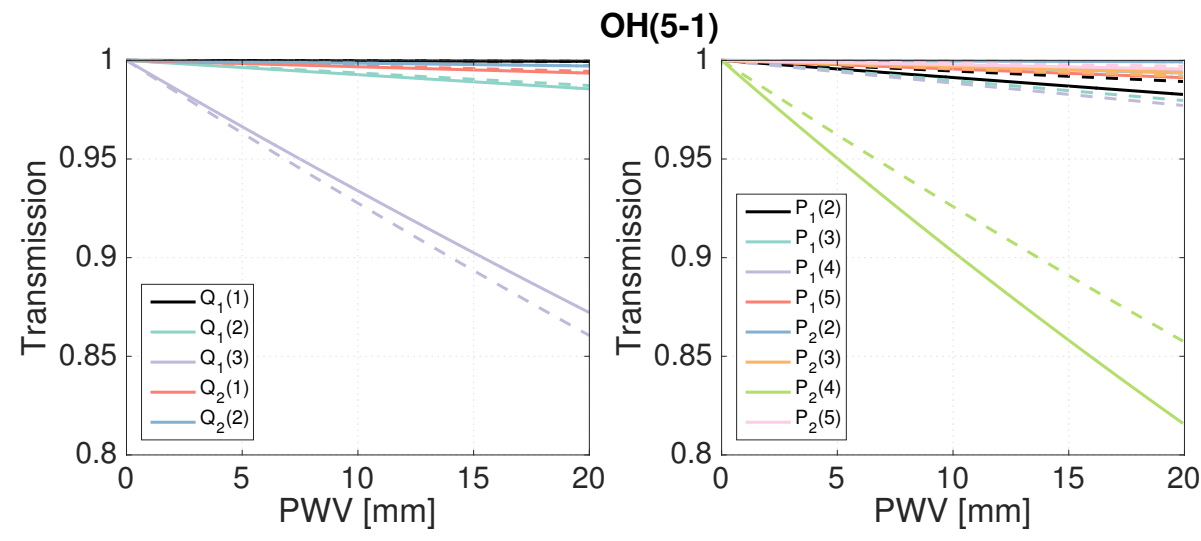

Figure 5. Water vapour transmission of the $\mathrm{OH}(5-1)$ lines as a function of precipitable water vapour (PWV). The left panel shows the Q-branch lines and the right panel, the P branch. Solid lines represent the e component of the lambda-doubled line, and the dashed lines represent the f component.

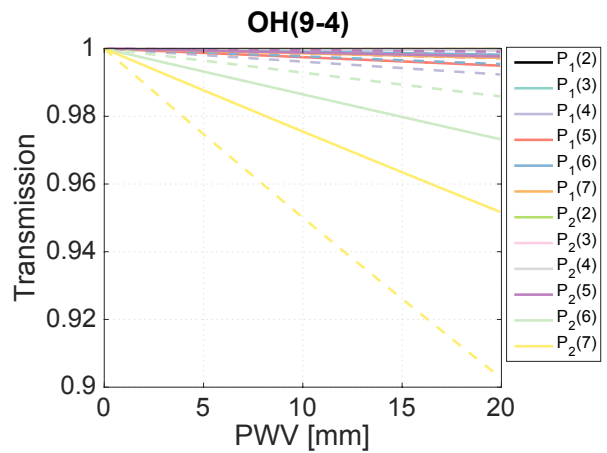

Figure 6. Water vapour transmission of the $\mathrm{OH}(9-4) \mathrm{P}-$ branch lines as a function of precipitable water vapour (PWV). Solid lines represent the e component of the lambda-doubled line, and the dashed lines represent the $\mathrm{f}$ component. The Q-branch transmissions are not represented for this band since they are all equal to 1 within $0.01 \%$ over the plotted range of PWV. in this study, carbon dioxide does not appear to be a major absorber.

\section{Hydroxyl temperatures}

To test the transmission values determined in this paper, we incorporate these into a calculation of $\mathrm{OH}(8-3)$ temperatures from spectral measurements from the HiTIES instrument. The resulting temperatures are plotted in Fig. 9. Twelve days of observations in December 2003 and January 2004 are used to obtain temperatures, using the method described in Sect. 2.2. The three strongest $\mathrm{P}$-branch lines are used $-\mathrm{P}_{1}(2)$, $\mathrm{P}_{1}(3)$, and $\mathrm{P}_{1}(4)$ - meaning three temperature estimates can be obtained for each measured spectrum, since one temperature determination requires the ratio of two $\mathrm{OH}$ line intensities, as per Eq. (4). Without correction for water vapour absorption, the three mean daily temperatures are different by 10 to $20 \mathrm{~K}$, similar to the discrepancies found by Phillips 


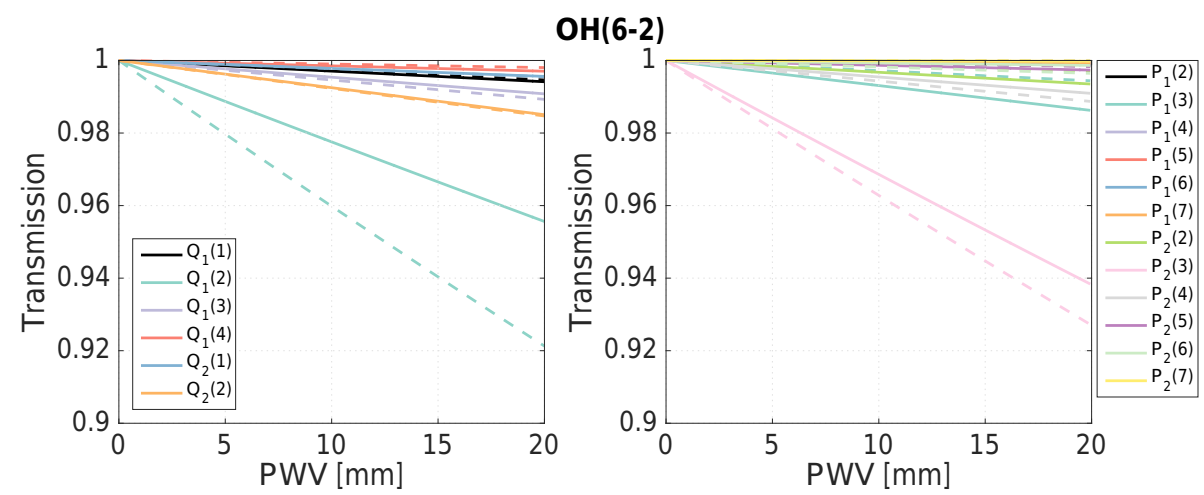

Figure 7. Water vapour transmission of the $\mathrm{OH}(6-2)$ lines as a function of precipitable water vapour (PWV). The left panel shows the Q-branch lines and the right panel, the P branch. Solid lines represent the e component of the lambda-doubled line, and the dashed lines represent the f component.
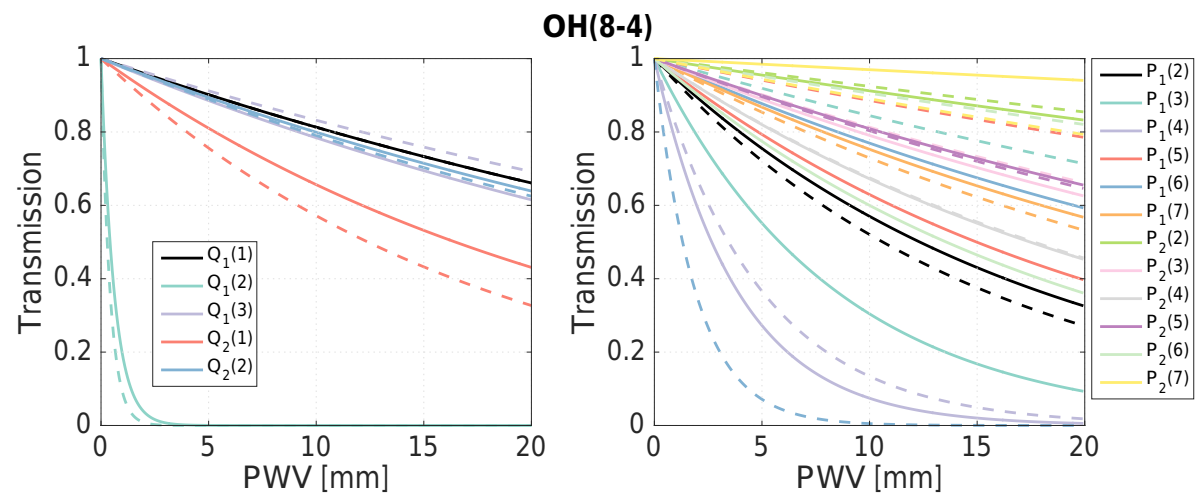

Figure 8. Water vapour transmission of the $\mathrm{OH}(8-4)$ lines as a function of precipitable water vapour (PWV). The left panel shows the Q-branch lines and the right panel, the P branch. Solid lines represent the e component of the lambda-doubled line, and the dashed lines represent the f component.

et al. (2004). This difference in temperature values is due to the absorption of the $\mathrm{P}_{1}(4)$ line by $\mathrm{H}_{2} \mathrm{O}$ (see Sect. 3). The daily mean temperatures without taking into account water vapour absorption are shown in dashed coloured lines in Fig. 9a: in blue for temperatures obtained by taking the ratio of $\mathrm{P}_{1}(2)$ and $\mathrm{P}_{1}(3)$, in red for $\mathrm{P}_{1}(2)$ and $\mathrm{P}_{1}(4)$, and in yellow for $\mathrm{P}_{1}(3)$ and $\mathrm{P}_{1}(4)$.

To correct for the absorption of $\mathrm{OH}$ emission by water vapour, we use the expressions and coefficients given in Table 1 for each of the P-branch lines used to determine temperatures. Since the three $\mathrm{OH}$ lines used are from the same vibrational band, the emission for each of them is produced at the same altitude. Thus, any difference in temperature estimation obtained from different ratios of these lines' intensity is assumed to be due only to differing amounts of water vapour absorption that their emission undergoes before reaching the HiTIES detector. Therefore, the column density of water vapour (or PWV) is chosen such that the three temperature determinations give the same result.

In this fashion, we obtain the temperatures and PWV values plotted in black in Fig. 9. Here we show only tempera- tures obtained during clear-sky periods, i.e. with no auroral emission present. Each temperature estimation (represented by a black point in Fig. 9a) is calculated with $\mathrm{OH}(8-3)$ line intensities measured from a spectrum composed of $20 \mathrm{~min}$ of HiTIES observations. The corresponding values of PWV for each of these temperature calculations are shown by the black points in Fig. 9b. The daily mean of all of these values is shown by the black solid lines in both panels.

The daily mean $\mathrm{OH}$ rotational temperature shows a variation of around $20 \mathrm{~K}$ over the course of a 2- to 3-day period. This is similar to what has previously been observed by Suzuki et al. (2009) and Espy et al. (2003) in $\mathrm{OH}$ airglow temperature measurements in Antarctica. These studies concluded that such rapid changes in $\mathrm{OH}$ rotational temperatures were due to changes in the meridional component of the mesospheric wind, caused by gravity waves. It is possible that such a mechanism is also responsible for the time variation of the $\mathrm{OH}$ temperatures that we measure here. Such changes in $\mathrm{OH}$ temperatures over a few days have also been seen in the Arctic (Nielsen et al., 2002; Dyrland et al., 2010; Holmen et al., 2014b). 

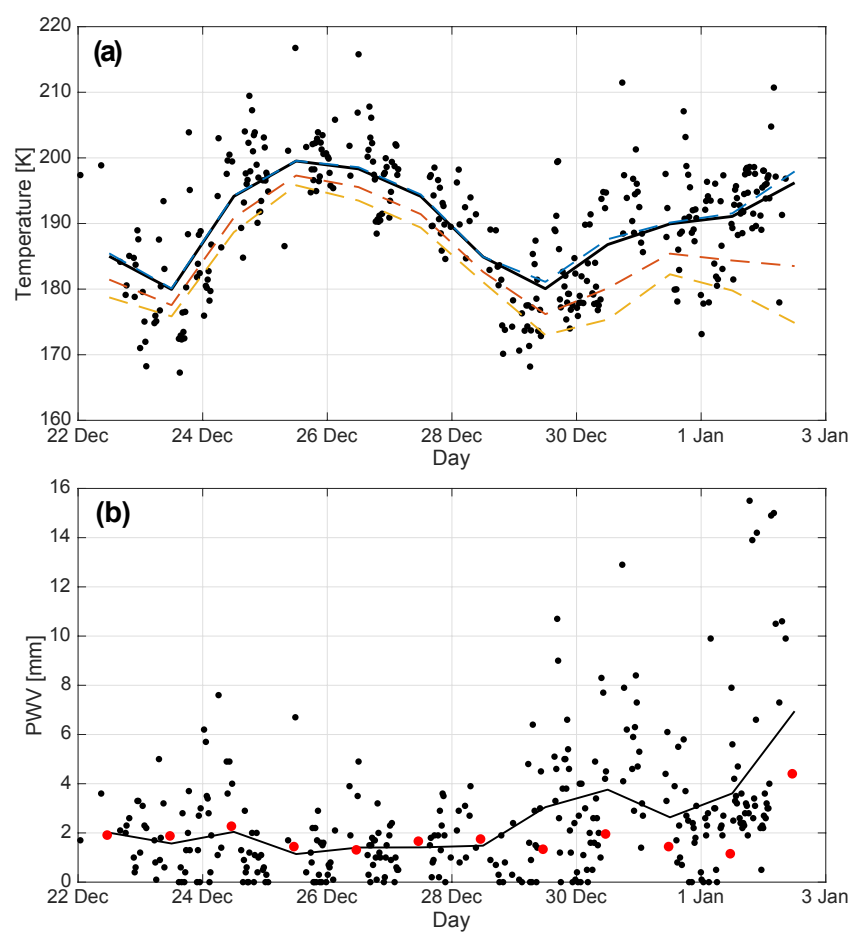

Figure 9. Panel (a): $\mathrm{OH}(8-3)$ temperatures obtained using $\mathrm{Hi}$ TIES. Each black point is obtained from a spectrum composed of $20 \mathrm{~min}$ of integration time. The solid black line is the daily mean. The coloured dashed lines represent the daily mean temperatures obtained when water vapour absorption is not taken into account. Temperatures obtained from the line ratio $\mathrm{P}_{1}(2) / \mathrm{P}_{1}(3)$ are shown in blue, those from $\mathrm{P}_{1}(2) / \mathrm{P}_{1}(4)$ are in red, and those from $\mathrm{P}_{1}(3) / \mathrm{P}_{1}(4)$ are in yellow. Panel (b): precipitable water vapour (PWV) obtained during each temperature calculation shown in panel (a), represented by black points. The daily mean of these is plotted in a solid black line. The red points represent PWV determined from the humidity profiles measured by the daily $\mathrm{Ny}$ Ålesund radiosonde measurements (Maturilli and Kayser, 2016). These data were recorded in December 2003 and January 2004.

In panel $b$ of Fig. 9, we compare the values of PWV obtained during the $\mathrm{OH}$ temperature determination (black points) with those calculated from the daily radiosonde measurements taken from Ny-Ålesund (Maturilli and Kayser, 2016). These radiosonde measurements match well the daily mean PWV obtained from HiTIES from 22 to 28 December. Thereafter, the HiTIES PWV values rise more steeply than those from the radiosonde. However, there is a corresponding large rise in the radiosonde PWV on 2 January 2004. The difference between the two measurements is not unexpected given that Ny-Ålesund and the site where HiTIES is located are $124 \mathrm{~km}$ apart, and that the sonde will drift in latitude and longitude as it rises with altitude and takes its measurements.

We also note the potential presence in the PWV estimations of a diurnal cycle, such as that seen by Dai et al. (2002) over North America. This trend in atmospheric water vapour content, as well as further study of $\mathrm{OH}$ temperatures obtained through the methods described in this paper, will be the topic of future studies.

\section{Conclusions}

We have modelled the absorption by water vapour of $\mathrm{OH}$ airglow emission in a number of different Meinel bands and tabulated the corresponding transmission coefficients. Water vapour absorption coefficients from the HITRAN2012 database were used. A number of $\mathrm{OH}$ vibrational bands have been chosen to apply this calculation to (8-3), (5-1), (9-4), (8-4), and (6-2); however, on request, we can determine water vapour absorption in other bands, as needed. Knowledge of these transmission coefficients allows the determination of accurate hydroxyl rotational temperatures.

Validation of the water vapour transmission coefficients in the $\mathrm{OH}(8-3)$ band has been carried out by applying them to 12 days of observations with the HiTIES instrument, located at the Kjell Henriksen Observatory, Svalbard. Without taking into account the effect of water vapour absorption on the $\mathrm{OH}$ lines, there was a discrepancy of about 10 to $20 \mathrm{~K}$ between temperatures obtained from different line intensity ratios. Using the transmission coefficients produced in this study allowed for this discrepancy to be eliminated.

A by-product of the method described in this paper is the possibility to obtain water vapour column densities. Using the HiTIES instrument, these can be determined at much higher cadence than using a radiosonde. Additionally, we always measure the same column of atmosphere, whereas a radiosonde will drift horizontally as it rises to take its measurements. Water vapour column densities determined from HiTIES observations in December 2003 have been shown to agree with values obtained from nearby radiosonde measurements.

For this study we have been limited to applying the water vapour transmission coefficients to the (8-3) band emission lines that have previously been measured with HiTIES. However, we have now developed a new mosaic filter which in future will allow $\mathrm{OH}(5-1)$ and $\mathrm{OH}(9-4)$ bands to be observed by the instrument. Future papers will further explore the trends in temperature and water vapour concentration that have been observed by HiTIES across different $\mathrm{OH}$ bands.

Data availability. Recent quick-look keograms from the Spectrographic Imaging Facility (SIF) are available at http://sif.unis.no. To access any data, please contact the authors.

Competing interests. The authors declare that they have no conflict of interest. 
Acknowledgements. J. M. Chadney, D. K. Whiter, and B. S. Lanchester are funded by the United Kingdom Natural Environment Research Council (NERC) under grant NE/N004051/1. The authors acknowledge the support of the Atmospheric Physics Group at University College London (UCL) in the operation of the Spectrographic Imaging Facility (SIF) and J. Sullivan, A. Stockton-Chalk, J. Holmes, and M. Dyrland in running the instruments during the winter campaign in 2003/4. We would also like to thank the staff at the University Centre in Svalbard (UNIS) for their support and the use of their facilities. The SIF was funded by the United Kingdom Particle Physics and Astronomy Research Council (PPARC) and NERC, and is a joint project between UCL and the University of Southampton.

The topical editor, C. Jacobi, thanks F. Sigernes and one anonymous referee for help in evaluating this paper.

\section{References}

Baker, D. J. and Stair, A. T.: Rocket measurements of the altitude distributions of the hydroxyl airglow, Phys. Scripta, 37, 611-622, doi:10.1088/0031-8949/37/4/021, 1988.

Chakrabarti, S., Pallamraju, D., Baumgardner, J., and Vaillancourt, J.: HiTIES: A High Throughput Imaging Echelle Spectrogragh for ground-based visible airglow and auroral studies, J. Geophys. Res., 106, 30337, doi:10.1029/2001JA001105, 2001.

Ciddor, P. E.: Refractive index of air: new equations for the visible and near infrared, Appl. Optics, 35, 1566, doi:10.1364/AO.35.001566, 1996.

Cosby, P. and Slanger, T.: OH spectroscopy and chemistry investigated with astronomical sky spectra, Can. J. Phys., 85, 77-99, doi:10.1139/P06-088, 2007.

Coxon, J. A. and Foster, S. C.: Rotational analysis of hydroxyl vibration-rotation emission bands: Molecular constants for $\mathrm{OH}$ $\mathrm{X} 2 \Pi, 6<=v<=10$, Can. J. Phys., 60, 41-48, doi:10.1139/p82$006,1982$.

Dai, A., Wang, J., Ware, R. H., and Van Hove, T.: Diurnal variation in water vapor over North America and its implications for sampling errors in radiosonde humidity, J. Geophys. Res.-Atmos., 107, ACL 11-1-ACL 11-14, doi:10.1029/2001JD000642, 2002.

Dyrland, M. E., Mulligan, F. J., Hall, C. M., Sigernes, F., Tsutsumi, M., and Deehr, C. S.: Response of $\mathrm{OH}$ airglow temperatures to neutral air dynamics at $78^{\circ} \mathrm{N}, 16^{\circ} \mathrm{E}$ during the anomalous 2003-2004 winter, J. Geophys. Res., 115, D07103, doi:10.1029/2009JD012726, 2010.

Espy, P. and Hammond, M.: Atmospheric transmission coefficients for hydroxyl rotational lines used in rotational temperature determinations, J. Quant. Spectrosc. Ra., 54, 879-889, doi:10.1016/0022-4073(95)00109-X, 1995.

Espy, P. J., Hibbins, R. E., Jones, G. O. L., Riggin, D. M., and Fritts, D. C.: Rapid, large-scale temperature changes in the polar mesosphere and their relationship to meridional flows, Geophys. Res. Lett., 30, 1240, doi:10.1029/2002GL016452, 2003.

Foucher, P. Y., Chédin, A., Armante, R., Boone, C., Crevoisier, C., and Bernath, P.: Carbon dioxide atmospheric vertical profiles retrieved from space observation using ACE-FTS solar occultation instrument, Atmos. Chem. Phys., 11, 2455-2470, doi:10.5194/acp-11-2455-2011, 2011.
French, W. J. R., Burns, G. B., Finlayson, K., Greet, P. A., Lowe, R. P., and Williams, P. F. B.: Hydroxyl (6-2) airglow emission intensity ratios for rotational temperature determination, Ann. Geophys., 18, 1293-1303, doi:10.1007/s00585-000-1293-2, 2000.

Holmen, S., Dyrland, M., and Sigernes, F.: Long-term trends and the effect of solar cycle variations on mesospheric winter temperatures over Longyearbyen, Svalbard $\left(78^{\circ} \mathrm{N}\right)$, J. Geophys. Res.Atmos., 119, 6596-6608, doi:10.1002/2013JD021195, 2014 a.

Holmen, S., Dyrland, M., and Sigernes, F.: Mesospheric temperatures derived from three decades of hydroxyl airglow measurements from Longyearbyen, Svalbard $\left(78^{\circ} \mathrm{N}\right)$, Acta Geophys., 62, 302-315, doi:10.2478/s11600-013-0159-4, 2014b.

Langhoff, S. R., Werner, H.-J., and Rosmus, P.: Theoretical transition probabilities for the $\mathrm{OH}$ meinel system, J. Mol. Spectrosc., 118, 507-529, doi:10.1016/0022-2852(86)90186-4, 1986.

Maturilli, M. and Kayser, M.: Arctic warming, moisture increase and circulation changes observed in the Ny-Ålesund homogenized radiosonde record, Theor. Appl. Climatol., 1-17, doi:10.1007/s00704-016-1864-0, 2016.

Meinel, A. B.: OH Emission Bands in the Spectrum of the Night Sky. I., Astrophys. J., 111, 555-564, doi:10.1086/145296, 1950a.

Meinel, A. B.: OH Emission Bands in the Spectrum of the Night Sky. II., Astrophys. J., 112, 120-130, doi:10.1086/145321, $1950 b$.

Mies, F. H.: Calculated vibrational transition probabilities of OH(Х2П), J. Mol. Spectrosc., 53, 150-188, doi:10.1016/00222852(74)90125-8, 1974.

Nielsen, K., Sigernes, F., Raustein, E., and Deehr, C.: The 20-year change of the Svalbard OH-temperatures, Phys. Chem. Earth, 27, 555-561, doi:10.1016/S1474-7065(02)00037-2, 2002.

Noll, S., Kausch, W., Kimeswenger, S., Unterguggenberger, S., and Jones, A. M.: OH populations and temperatures from simultaneous spectroscopic observations of 25 bands, Atmos. Chem. Phys., 15, 3647-3669, doi:10.5194/acp-15-3647-2015, 2015.

Noll, S., Kausch, W., Kimeswenger, S., Unterguggenberger, S., and Jones, A. M.: Comparison of VLT/X-shooter $\mathrm{OH}$ and $\mathrm{O} 2$ rotational temperatures with consideration of TIMED/SABER emission and temperature profiles, Atmos. Chem. Phys., 16, 50215042, doi:10.5194/acp-16-5021-2016, 2016.

Perminov, V. I., Semenov, A. I., and Shefov, N. N.: On rotational temperature of the hydroxyl emission, Geomagn. Aeronomy, 47, 756-763, doi:10.1134/S0016793207060084, 2007.

Phillips, F., Burns, G. B., French, W. J. R., Williams, P. F. B., Klekociuk, A. R., and Lowe, R. P.: Determining rotational temperatures from the $\mathrm{OH}(8-3)$ band, and a comparison with $\mathrm{OH}(6-2)$ rotational temperatures at Davis, Antarctica, Ann. Geophys., 22, 1549-1561, doi:10.5194/angeo-22-1549-2004, 2004.

Rothman, L., Gordon, I., Babikov, Y., Barbe, A., Chris Benner, D., Bernath, P., Birk, M., Bizzocchi, L., Boudon, V., Brown, L., Campargue, A., Chance, K., Cohen, E., Coudert, L., Devi, V., Drouin, B., Fayt, A., Flaud, J.-M., Gamache, R., Harrison, J., Hartmann, J.-M., Hill, C., Hodges, J., Jacquemart, D., Jolly, A., Lamouroux, J., Le Roy, R., Li, G., Long, D., Lyulin, O., Mackie, C., Massie, S., Mikhailenko, S., Müller, H., Naumenko, O., Nikitin, A., Orphal, J., Perevalov, V., Perrin, A., Polovtseva, E., Richard, C., Smith, M., Starikova, E., Sung, K., Tashkun, S., Tennyson, J., Toon, G., Tyuterev, V., and Wagner, G.: The HITRAN2012 molecular spectroscopic database, J. Quant. Spectrosc. Ra., 130, 4-50, doi:10.1016/j.jqsrt.2013.07.002, 2013. 
Rousselot, P., Lidman, C., Cuby, J. G., Moreels, G., and Monnet, G.: Night-sky spectral atlas of $\mathrm{OH}$ emission lines in the nearinfrared, Astron. Astrophys., 354, 1134-1150, 2000.

Sharpee, B. D., Slanger, T. G., Huestis, D. L., and Cosby, P. C.: Measurements of the Singly Ionized Oxygen Auroral Doublet Lines $\lambda \lambda 7320,7330$ Using High-Resolution Sky Spectra, Astrophys. J., 606, 605-610, doi:10.1086/382869, 2004.

Sigernes, F., Shumilov, N., Deehr, C. S., Nielsen, K. P., Svenøe, T., and Havnes, O.: Hydroxyl rotational temperature record from the auroral station in Adventdalen, Svalbard $\left(78^{\circ} \mathrm{N}, 15^{\circ} \mathrm{E}\right), \mathrm{J}$. Geophys. Res., 108, 1342, doi:10.1029/2001JA009023, 2003.

Suzuki, H., Taguchi, M., Kanai, Y., and Takeyama, N.: Fast spectrometer for ground-based observations of $\mathrm{OH}$ rotational temperature, Appl. Optics, 48, 1119, doi:10.1364/AO.48.001119, 2009.

Suzuki, H., Tsutsumi, M., Nakamura, T., and Taguchi, M.: The increase in $\mathrm{OH}$ rotational temperature during an active aurora event, Ann. Geophys., 28, 705-710, doi:10.5194/angeo-28-7052010, 2010.
Turnbull, D. and Lowe, R.: New hydroxyl transition probabilities and their importance in airglow studies, Planet. Space Sci., 37, 723-738, doi:10.1016/0032-0633(89)90042-1, 1989.

von Savigny, C., McDade, I. C., Eichmann, K.-U., and Burrows, J. P.: On the dependence of the $\mathrm{OH}^{*}$ Meinel emission altitude on vibrational level: SCIAMACHY observations and model simulations, Atmos. Chem. Phys., 12, 8813-8828, doi:10.5194/acp-128813-2012, 2012.

Whiter, D. K., Lanchester, B. S., Gustavsson, B., Jallo, N. I. B., Jokiaho, O., Ivchenko, N., and Dahlgren, H.: Relative brightness of the $\mathrm{O}^{+}\left({ }^{2} \mathrm{D}-{ }^{2} \mathrm{P}\right)$ doublets in low-energy aurorae, Astrophys. J., 797, 64-72, doi:10.1088/0004-637X/797/1/64, 2014. 